\title{
A Comparative Study of Single-Dose Treatment of Chancroid Using Thiamphenicol versus Azithromycin
}

\begin{abstract}
Walter B. Junior ${ }^{1}$, Nilton G. Di Chiacchio ${ }^{2}$, Nilton Di Chiacchio ${ }^{2}$, Ricardo Romiti ${ }^{1}$, Paulo R. Criado ${ }^{3}$ and Paulo Eduardo N. Ferreira Velho ${ }^{4}$ ${ }^{1}$ Department of Dermatology, São Paulo University; ${ }^{2}$ Santo Amaro University; ${ }^{3}$ Division of Dermatology, Hospital das Clinicas, São Paulo University; ${ }^{4}$ Division of Dermatology, Faculty of Medical Sciences, State University of Campinas (UNICAMP); São Paulo, Brazil
\end{abstract}

\begin{abstract}
A study was conducted in São Paulo, Brazil, to compare azithromycin with thiamphenicol for the single-dose treatment of chancroid. In all, 54 men with chancroid were tested. The etiology was determined by clinical characterization and direct bacterioscopy with Gram staining. None of the patients had positive serology or darkfield examination indicating active infection with Treponema pallidum. Genital infections due to Neisseria gonorrhoeae and herpes simplex virus were excluded by polymerase chain reaction testing. For 54 patients with chancroid, cure rates with single-dose treatment were $73 \%$ with azithromycin and $89 \%$ with thiamphenicol. HIV seropositivity was found to be associated with treatment failure $(p=0.001)$. The treatment failed in all HIV positive patients treated with azithromycin $(p=0.002)$ and this drug should be avoided in these co-infected patients. In the view of the authors, thiamphenicol is the most indicated single-dose regimen for chancroid treatment.

Key-Words: Azithromycin, chancroid, Haemophilus ducreyi, single dose, thiamphenicol.
\end{abstract}

Chancroid is a sexually transmitted infection caused by the Gram negative bacterium Haemophilus ducreyi. The disease is manifested as genital ulceration which may be accompanied by regional lymphadenitis and bubo formation. Chancroid may also be spread to other anatomical sites by auto-inoculation, a clinical feature first demonstrated experimentally by Ducrey in 1889 [1]. It occurs sporadically in the developed world, usually in individuals who have recently returned from chancroid endemic areas or occasionally within the context of localized urban outbreaks which may be associated with commercial sex workers [2-4]. Within the resource poor regions of the world, however, chancroid remains an important cause of genital ulceration syndrome [5].

Genital ulceration has been shown to be a major co-factor in the transmission of human immunodeficiency virus type 1 (HIV-1) infection both through cross sectional cohort studies and prospective longitudinal studies [6,7]. Therefore, effective diagnosis and treatment of chancroid may play an important role in slowing down the HIV-1 epidemic in those parts of the world where both diseases are prevalent $[4,8]$.

Treatment options for chancroid have become increasingly limited because of the development of resistance to several available and affordable antimicrobial medicines $[9,10]$, which has led to the search for an alternative treatment regimen for chancroid.

The World Health Organization (WHO) recommends a seven-day course of erythromycin administered orally as a first line treatment for chancroid. Although effective, poor compliance and gastro-intestinal intolerance make alternative therapy desirable. Other options that are presented as first line treatments are 500mg of ciprofloxacin administered twice

Received on 20 February 2009; revised 6 May 2009.

Address for correspondence: Dr. Walter Belda Junior. Av. Açocê, 162, Moema, São Paulo, Brazil. Zip code: 04075-020. Phone. 55 (11) 50511921. Fax: 55 (11) 50515141. E-mail: walterbelda@uol.com.br.

The Brazilian Journal of Infectious Diseases 2009;13(3):218-220. (C) 2009 by The Brazilian Journal of Infectious Diseases and Contexto Publishing. All rights reserved. a day for 3 days or a single-dose of $1 \mathrm{~g}$ of azythromycin [11]. Since the advent of the antimicrobial era, single-dose therapy has been a valuable tool in the management of genital infection. Most of the common sexually transmitted diseases, such as gonococcal and non-gonococcal urethritis, primary syphilis and trichomoniasis, can be treated in this way, as can genital infections which are not sexually transmitted, such as bacterial vaginosis and genital tract candidiasis [5,12].

The main advantages of single-dose therapy are convenience, its ability to ensure virtually $100 \%$ compliance, its potentially lower cost, and the possibility of reducing the emergence of resistance [4,5].

Azithromycin is an effective oral therapy for chancroid and other sexually transmitted disease pathogens [11], but its high cost makes it less suitable in financially challenged settings $[4,13,14]$, as compared with thiamphenicol $[15,16]$.

\section{Material and Methods}

An open, prospective, comparative, non-blind cohort study which was conducted was designed to evaluate the efficiency and safety of two treatment plans for chancroid and the effect of HIV-1 infections on treatment response.

The study was conducted between November, 2005, and September, 2007, at the Hospital das Clinicas of São Paulo University. Sixty patients with necrotising genital ulcerations on their prepuces and frenula were received at the sexually transmitted diseases clinic. Only patients with purulent ulcers assessed to be clinically compatible with chancroid were enrolled. In addition, all patients had to be over 18 years of age and willing to return to the clinic for follow-up. After determining eligibility, a formal explanation of protocol was provided to each participant. The participants were also tested for HIV.

The diagnoses were confirmed by clinical characterization of genital necrotic and painful chancres. A direct bacterioscopy with Gram staining was conducted which revealed Gramnegative bacillus forming palisades or simple chains in a typical disposition in the polymorphonuclear cytoplasm, on material obtained from chancre pus. 
None of the patients had positive serology or dark-field examinations indicating active infection with Treponema pallidum. Genital infections due to Neisseria gonorrhoeae and herpes simplex virus were excluded by polymerase chain reaction testing.

As soon as the clinical laboratory diagnosis was established, the patients each received $5 \mathrm{~g}$ of granulated thiamphenicol dissolved in $50 \mathrm{~mL}$ of water in a single-dose or $1 \mathrm{~g}$ of azithromycin in a single-dose. The medication was taken under direct supervision.

Three patients in the thiamphenicol group and four patients in the azithromicyn group had concomitant infection with human immunodeficiency virus type 1 (HIV-1).

Weekly follow-up visits were arranged until complete cure had occurred. It was established as complete re-epithelisation of the ulcers at the $21^{\text {th }}$ days of follow-up. Ineffective treatment was determined if the lesions were still ulcerated at that time and with they presented positive bacterioscopy, in which case rescue treatment was prescribed. The rescue treatment consisted of a 7-10 day course of erythromycin.

The Fisher test was used to compare groups.

\section{Results}

Of the 60 patients initially invited to participate in the evaluation, 54 subsequently returned for follow-up examinations. Of the 54 available patients included in the study, 26 received treatment with azithromycin and 28 with thiamphenicol (Table 1).

For most of the patients, the ulcers improved symptomatically within four to six days and substancial re-epithelisation occurred within nine days after the onset of therapy. The time required for complete healing was ten days in HIV negative patients. Ulcers in HIV-1 positive patients treated with thiamphenicol re-epithelized after two weeks.

Overall, cure rates of $73 \%$ and $89 \%$ were recorded following treatment with azithromycin and thiamphenicol, respectively. There were seven clinical failures in the azithromycin group and three in the thiamphenicol group. All of them had a positive bacterioscopy from the ulcer during the follow-up period. Only three patients from the azithromycin group and two of ten patients who needed erythromycin treatment reported mild abdominal discomfort.
The treatment failed in all HIV positive patients treated with azithromycin $(\mathrm{p}=0.002)$ and in one HIV positive patient treated with thiamphenicol. HIV seropositivity was found to be associated with treatment failure $(\mathrm{p}=0.001)$.

\section{Discussion}

Recommended and alternative treatment regimens from WHO, the Centers for Disease Control and Prevention (CDC), the United Kingdom's Clinical Effectiveness Group, and the Brazilian Health Ministry included 500mg of erythromycin three or four times a day for seven days, 500mg of ciprofloxacin twice a day for 3 days, $1 \mathrm{~g}$ of azithromycin single-dose or $250 \mathrm{mg}$ of ceftriaxone single-dose intra-muscular. The United Kingdom's Clinical Effectiveness Group also recommended 500mg of ciprofloxacin as a single-dose regimen [11,17-19]. Erythromycin has an inconvenient multi-dosing regimen.

Thiamphenicol (dextrosulfenidol), synthesized in 1951, belongs to the aminic derivatives of the hydrocarbilsulfonylpropandiol group, and is obtained from methylcarptoacetophenone. The similarity between the chemical structure of thiamphenicol and chloramphenicol has raised questions regarding the possible development of aplastic anemia. Toxic effects of chloramphenicol are caused by the nitro group. The fundamental difference between chloramphenicol and thiamphenicol is the presence in the later agent of the methylsulfonic radical in place of the nitro group, and the absence of this group in thiamphenicol probably accounts for the reduced risk associated with its use. At a sub-cellular level, thiamphenicol inhibits protein synthesis, joining the ribosomes and then preventing the binding of aminoacids with peptidyl transferase $[15,20]$.

Treatment results suggested that $5 \mathrm{~g}$ of thiamphenicol is more effective than a single-dose treatment of azithromicyn for chancroid, even though there is no statistical significance in the comparison. Among the 28 patients given thiamphenicol, only three failed to respond to treatment (one of whom was co-infected with HIV-1). Azithromycin also performed well in 19 patients and failed in seven (four of whom were co-infected with HIV-1) and should be avoided in HIV-1 positive patients $(\mathrm{p}=0.002)$. All chancroid patients who were placed on rescue therapy responded to treatment with erythromycin. The two treatment regimens were welltolerated.

Table 1. Azythromycin and thiamphenicol treatments for patients with and without HIV co-infection.

\begin{tabular}{llcrc}
\hline & & Cure & Total & p* \\
\hline Treatment independent & HIV+ & $2(28.5 \%)$ & 7 & $0.001^{* *}$ \\
& HIV- & $42(89.3 \%)$ & 47 & \\
Azithromycin & HIV+ & $0(0.0 \%)$ & 4 & $0.002^{* *}$ \\
\multirow{3}{*}{ Thiamphenicol } & HIV- & $19(86.3 \%)$ & 22 & 0.297 \\
& HIV+ & $2(66.6 \%)$ & 3 & \\
\hline
\end{tabular}

* p to Fisher test. ** Significant difference to confidence interval of $95 \%$. 
This study showed that HIV-1 infection alters the response to treatment $(\mathrm{p}=0.001)$.

\section{Conclusion}

Chancroid remains an important cause of genital ulceration syndrome in the tropics where erythromycin has been the mainstay of therapy. Single-dose oral regimens of azithromycin or thiamphenicol offer advantages in terms of improving patients' compliance. The study showed that HIV seropositive patients are more likely to fail single-dose therapeutic regimens, which is an indication that these groups of patients need more intensive follow-up, as was suggested earlier [4,14,18,21]. In the view of the authors, thiamphenicol is the most indicated single-dose regimen for chancroid treatment since results suggest that azythromycin should be avoided in HIV positive patients.

\section{References}

1. Belda, Jr. W., Siqueira L.F.G. Cancro Mole. In: Belda, Jr. W (ed). Doença Sexualmente Transmissível. São Paulo: Editora Atheneu; 1999, pp. 47-52.

2. Hammond G.W., Slutchuk M., Scatliff J. Epidemiologic, Clinical Laboratory and Therapeutic Features of an Urban Outbreak of Chancroid in North America. Rev Infect Dis 1980;2: 867-79.

3. DiCarlo R.P., Armentor B.S., Martin D.H. Chancroid Epidemiology in New Orleans Men J Infect Dis 1995;172:446-52.

4. Mohammed T.T., Olumide Y.M. Chancroid and Human Immunodeficiency Virus Infection-A Review. Int J Dermatol 2008;47(1):1-8.

5. Annan N.T., Lewis D.A. Treatment of Chancroid in ResourcePoor Countries. Expert Rev Anti Infec Ther 2005;3(2):295306.

6. Fleming D.T., Wasserhert J.N. From Epidemiological Synergy to Public Health Policy and Practice: The Contribution of Other Sexually Transmitted Diseases to Sexual Transmission of HIV Infection. Sex Transm Infect 1999;75:3-17.

7. Plummer F.A., Simonsen J.N., Cameron D.W. Cofactors in MaleFemale Sexual Transmission of Human Immunodeficiency Virus Type 1. J Infect Dis 1991;163:233-9.
8. Lewis D.A. Chancroid: Clinical Manifestation, Diagnosis and Management. Sex Transm Infect 2003;79:68-71.

9. Aldrige K.E., Cammarata C., Martin D.H. Comparison of the in vitro Activities of Various Parenteral and Oral Antimicrobial Agents against Endemic Haemophilus ducreyi. Antimicrob Agents Chemother 1992;36:1639-43.

10. Rutanarugsa A., Vorachit M., Polmikorn N., Jayanetra P. Drug Resistance of Haemophilus ducreyi. Southeast Asian J Trop Med Public Health 1990;21:185-93.

11. World Health Organization. Global Strategy for the Prevention and Control of Sexually Transmitted Infections: 2006-2015. p. 6 Geneva, Switzerland, 2007.

12. Kingston M., Carlin E. Treatment of Sexually Transmitted Infections with Single Dose Therapy: A Double-edged Sword. Drugs 2002;62:871-8.

13. Martin D.H., Sargent S., Wendel G., McCormak W., Spier N., Johnson R. Comparison of Azithromycin and Ceftriaxone for the Treatment of Chancroid. Clin Infect Dis 1995;21:409-19.

14. Tyndall M.W., Agoki E., Plummer F., Malisa W., Ndinya-Achola J.U., Ronald A.R. Single Dose Azithromycin for the Treatment of Chancroid: A Randomized Comparison with Erythromycin. Sex Transm Dis 1994;21:231-4.

15. Belda, Jr. W., Siqueira L.F.G., Fagundes L.J. Thiamphenicol in the Treatment of Chancroid. A Study of 1.128 Cases. Rev Inst Med trop São Paulo 2000;42:133-5.

16. Belda, Jr. W., Velho P.E.N.F., Arnone M., Romitti R. Donovanosis Treated with Thiamphenicol. Br J Infect Dis 2007;11(4):388-9.

17. British Association for Sexual Health and HIV (BASSH). National Guideline for the Management of Chancroid. Clinical Effectiveness Group. http://www.bashh.org/guidelines/2007/Chancroid_2007.pdf.

18. U.S. Department of Health and Human Services. Centers for Disease Control and Prevention. Chancroid in Sexually Transmitted Diseases Treatment Guidelines. MMWR. 2006;55(11):20-1.

19. Brasil, Ministério da Saúde, Secretaria de Vigilância em Saúde, Programa Nacional de DST/AIDS. Donovanose in Manual de Controle de Doenças Sexualmente Transmissíveis: DST. $4^{\mathrm{a}}$ ed. Série Manuais $\mathrm{n}^{\circ}$ 68, p. 50. Brasília, DF; 2006.

20. Belda W., Santos, Jr. M.F.Q., Belda, Jr. W. Thiamphenicol in the Treatment of Male Gonococcal Urethritis. A study of 1.230 Cases. Sex Transm Dis 1984;11:418-9.

21. Ballard R.C., Ye H., Matta A., Dangor Y., Radebe F. Treatment of Chancroid with Azithromycin. Int J STD AIDS 1996;7(suppl 1):S9-S12. 